\title{
The sigmoid colon and bladder shielding in whole pelvic irradiation at prostate cancer (forward planned IMRT from Institute of Oncology Ljubljana)
}

\author{
Daša Grabec ${ }^{1}$ and Borut Kragelj ${ }^{2}$ \\ ${ }^{1}$ Radiophysics Unit, ${ }^{2}$ Department of Radiotherapy, Institute of Oncology Ljubljana, \\ Ljubljana, Slovenia
}

\begin{abstract}
Background. The whole pelvic irradiation (WPI) is again gaining the important role in radiotherapy of prostate cancer. With the conformal irradiation of the large fields that are covering the pelvic nodes, the extra damage is done to sigmoid colon and urinary bladder. Sparing the region between the iliac nodes is necessary since the dose delivered to the organs at risk that are partly included in the mentioned area (the sigmoid colon and bladder) can be importantly reduced.

Methods. We are presenting a possible way of shielding the central region between iliac nodes that does not need to be irradiated. Dose volume histograms of standard box technique and technique with additional central shielding of sigmoid colon and urinary bladder in WPI are compared in 10 patients.

Results. Applying the described shielding technique 30 to $45 \%$ or in some cases even up to $55 \%$ of the sigmoid colon that would with standard box technique be irradiated at doses from 30 to 50 Gy is spared, and also around 10\% of the bladder that would receive 45-55 Gy is spared.

Conclusions. In the whole pelvic irradiation (WPI) in prostate radiotherapy the sparing of the region between the iliac nodes is crucial since it allows the dose delivered to the sigmoid colon to be reduced to the recommended restrictions. Since the sigmoid colon benefits most from described shielding, the technique is addressed as the Sigmoid Colon Shielding (SCS). The SCS technique is in use at our department at Institute of Oncology in Ljubljana.
\end{abstract}

Key words: prostate radiotherapy; whole pelvic irradiation; sigmoid colon shielding; dose distribution; forward planned IMRT

\section{Introduction}

Received 29 November 2008

Accepted 9 December 2008

Correspondence to: Daša Grabec, PhD, Radiophysics Unit, Department of Radiotherapy, Institute of Oncology Ljubljana, Zaloška 2, SI-1000 Ljubljana, Slovenia. Phone: +386 1 5225238; Fax: +386 14319108 ; E-mail: dgrabec@onko-i.si
The whole pelvic irradiation (WPI) is again gaining the important role in radiotherapy of prostate cancer. ${ }^{1,2}$ The radiotherapy for locally advanced and high risk localized prostate tumour at our department consists 
of the treatment of three planning target volumes (PTV1, PTV2 and PTV3) irradiated in 42 fractions $(28+5+9)$ of 180 cGy.

PTV1 represents the WPI that is irradiated up to $50.4 \mathrm{~Gy}$. PTV1 includes prostate, seminal vesicles and nodes with specific margins.

Gross target volume $(\mathrm{GTV})=$ prostate

Clinical target volume $1(\mathrm{CTV} 1)=\mathrm{GTV}+$ CTV seminal vesicles + CTV Nodes

PTV1 $=(\mathrm{GTV}+$ Pmargin $)+(\mathrm{CTV} \mathrm{sv}+$ svmargin $)+(\mathrm{CTV} N+$ Nmargin $)$

Nodes that are included in CTV $\mathrm{N}$ are pelvic nodes in the region of iliacae communis, nodes around iliacae externe and interne in the length of 7 to $8 \mathrm{~cm}$ from iliac vessels bifurcation and nodes around aortic bifurcation. Perirectal nodes are not included in CTV $\mathrm{N}$ due to the otherwise unacceptable toxicity on rectum. ${ }^{3,4}$ To lower the dose on rectum and sigmoid colon and due to the low incidence of the appearance in the area, ${ }^{5}$ presacral nodes are not entirely included in CTV N. Margins of PTV1 are uniform $1 \mathrm{~cm}$, but are also reduced when necessary in order to attain the treatment plan that respects the restrictions on rectum.

PTV2 and PTV3 represent the boost on the seminal vesicles and prostate. PTV2 that includes prostate with seminal vesicles is irradiated up to 59.4 Gy and PTV3 which is boost on prostate alone and is finally irradiated up to $75.6 \mathrm{~Gy}$. The boost treatment delivery is image guided. Prior to the treatment planning three golden markers are implanted in the patient's prostate, and the boost treatment fields of PTV2 and PTV3 are positioned according to the marker locations. Due to the image guiding the dorsal prostate margins (PBmargin) in PTV2 and PTV 3 are shrunk to $7 \mathrm{~mm}$.

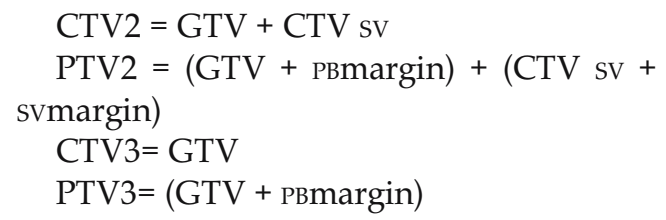

With treatment planning we are trying to spare organs at risk (OR) and tend to assure the restrictions on OR. With the restrictions we are applying the irradiation toxicity on OR are still acceptable:3,4

Rectum: V50<64\%, V60<45\%, V70<25\%, Dmean<45 Gy,

Anus: V55<16\%, V60<5\%, Dmean $<$ 40Gy.

In our experience, we found out that those restrictions could not be achieved when perirectal nodes were included in CTV N. Cost - benefit calculation lead us to the solution to exclude perirectal nodes from CTV N.

For sigmoid colon we aim to apply the same restrictions that are in use for rectum. ${ }^{6,7}$

We are also respecting that more than half of urinary bladder should not receive doses higher than 70 Gy (V70<50\%). Since the stricter dose constraints on bladder, that prevent G1 and G2 toxicity, ${ }^{8,9}$ cannot be respected, applying WPI, the chosen restrictions are set to prevent G3 toxicity.

We are checking also the dose on hips and penile bulb, but we are not altering the treatment due to the high doses on hips and penile bulb.

The planning target volumes PTV1, PTV2 and PTV3 are required to be enclosed in 95\% isodose relative to the prescribed dose. The maximal dose should not exceed $107 \%$ of the prescribed dose.

\section{The treatment plan}

With the conformal irradiation with box technique (4 fields of gantries: $0^{\circ}, 90^{\circ}$, $180^{\circ}, 270^{\circ}$ ) and smaller fields of mentioned gantries (forward planned IMRT), the dose is delivered to the large volume PTV1. The smaller fields added in order to homogenize the delivered dose, ${ }^{10}$ are usually applied in the region of prostate, where the dose delivered with the bigger fields 


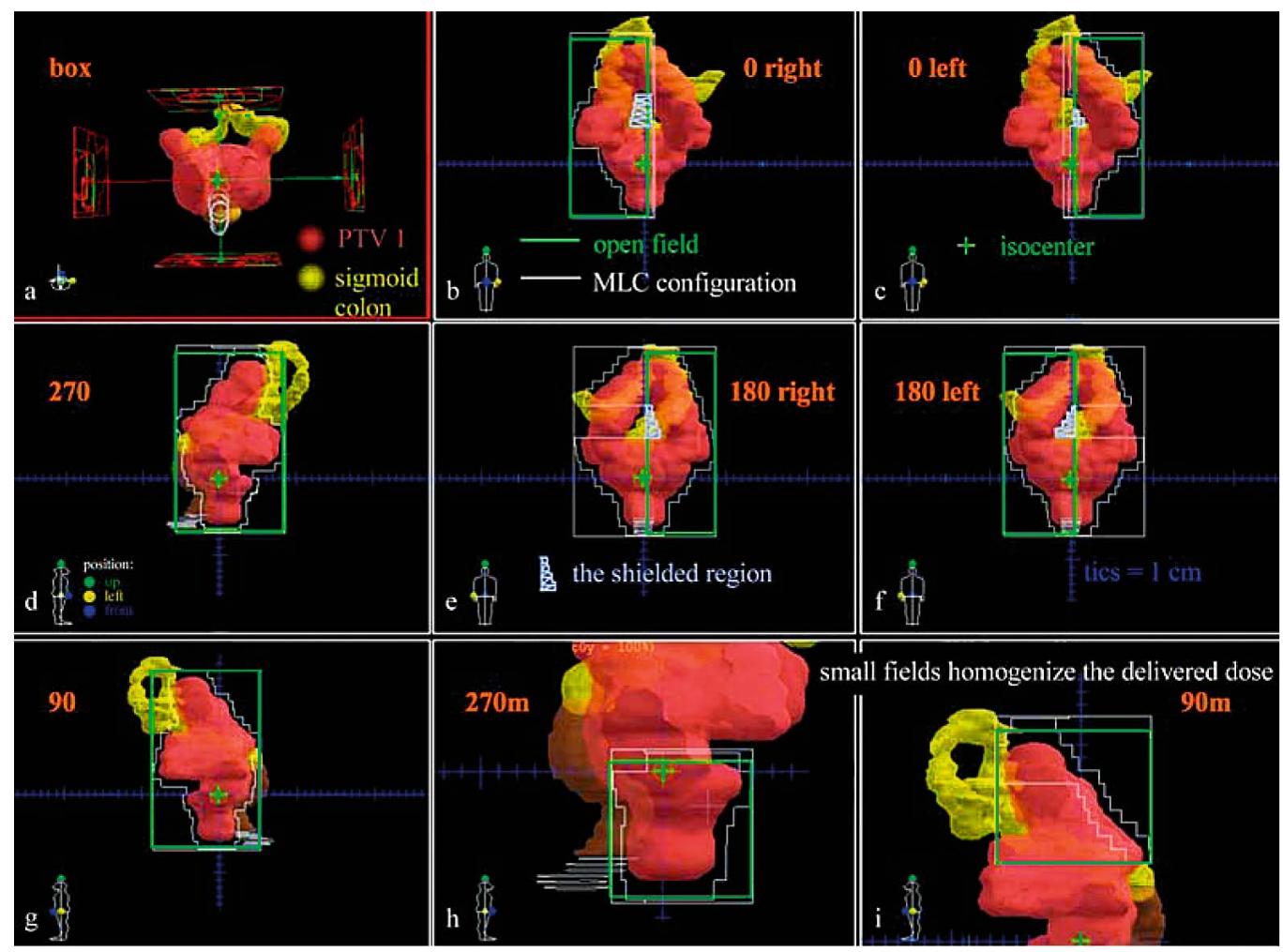

Figure 1. The sigmoid colon shielding (SCS) technique. The classical box technique consists of the four fields of gantries: $0^{\circ}, 90^{\circ}, 180^{\circ}$ and $270^{\circ}$, shaped to the planning target volume (PTV) (a). The fields are named according their gantries. In SCS technique the box technique is altered so the central part that is not included in the PTV1 can be shielded. In SCS the irradiation gantries remain the same as with classical box technique. In order to shield the central part fields $0^{\circ}$ and $180^{\circ}$ have to be split through the region of shielding. Right and left contributions to the irradiation from $0^{\circ}$ are presented on $\mathrm{b}$ and $\mathrm{c}$, whereas e and $\mathrm{f}$ present the right and left contributions from $180^{\circ}$. Presented fields $0^{\circ}$ and $180^{\circ}$ are off centre split $\left(0^{\circ}: 0.5 \mathrm{~cm}\right.$ to the left from the isocentre, $180^{\circ}: 0.5 \mathrm{~cm}$ to the right from the isocentre). The collimator is set to $0^{\circ}$, so the MLC can slide in the region that needs to be shielded. The outer margins of the fields remain the same as the ones the primary field. The part of sigmoid colon (yellow) in the shielded region is spared. Lateral irradiation remain intact, major fields $270^{\circ}$ and $90^{\circ}(\mathrm{d}, \mathrm{g})$, as well as the small fields that homogenize the dose distribution $270^{\circ} \mathrm{m}$ and $90^{\circ} \mathrm{m}$ (h and i).

is not sufficient, due to the bony anatomy and due to the patient's shape. With the dose delivered to the large fields of PTV1, the dose is delivered also to the region between the iliac nodes that does not need to be irradiated. Keep at box technique, the dose delivered to the region between iliac nodes, that is not included in PTV1 could be reduced, by shielding pieces of the large fields from $0^{\circ}$ and $180^{\circ}$.

Considering the uniform dose delivery from all four sides of the box, the dose to the shielded region can be lowered for $50 \%$. Since, as we are describing later on, the sigmoid colon benefits most from such shielding, the described technique is addressed as the Sigmoid Colon Shielding (SCS). It is important to pay attention on sigmoid colon since it appears that dose on sigmoid colon is co-responsible for lower intestinal toxicity. ${ }^{7}$ At our department we achieve the SCS in different ways: with individual shielding blocks or with the "off centre field splitting". All the changes required for SCS re- 
Table 1. The average DVH parameters for sigmoid colon and bladder. The average DVH parameters with corresponding standard deviations are presented for sigmoid colon and bladder. For the every patient the DVH parameters were calculated for BOX and for SCS technique as well as for the difference between techniques (BOX - SCS). The average DVH parameters and corresponding standard deviations were calculated from 10 consecutive patients. Even though the number of patients is small the clear advantage of the SCS technique is visible from the comparison of the DVH parameters. The beneficial DVH parameters changes that are higher than the standard deviations are indicated with yellow.

\begin{tabular}{|c|c|c|c|c|c|c|c|c|}
\hline Organ\& technique & $\begin{array}{l}\text { V30 } \\
{[\%]}\end{array}$ & $\begin{array}{l}\text { V35 } \\
{[\%]}\end{array}$ & $\begin{array}{l}\text { V40 } \\
{[\%]}\end{array}$ & $\begin{array}{l}\text { V45 } \\
{[\%]}\end{array}$ & $\begin{array}{l}\text { V50 } \\
{[\%]}\end{array}$ & $\begin{array}{l}\text { V55 } \\
{[\%]}\end{array}$ & $\begin{array}{l}\text { V60 } \\
{[\%]}\end{array}$ & $\begin{array}{l}\text { Dmean } \\
\text { [Gy] }\end{array}$ \\
\hline $\begin{array}{l}\text { sigmoid colon } \\
\text { BOX }\end{array}$ & $\begin{array}{l}90.2 \\
\pm 16.67\end{array}$ & $\begin{array}{l}86.36 \\
\pm 17.35\end{array}$ & $\begin{array}{l}82.61 \\
\pm 17.51\end{array}$ & $\begin{array}{l}78.61 \\
\pm 17.65\end{array}$ & $\begin{array}{l}72.66 \\
\pm 17.91\end{array}$ & $\begin{array}{l}9.54 \\
\pm 9.44\end{array}$ & $\begin{array}{l}4.36 \\
\pm 4.97\end{array}$ & $\begin{array}{l}47.97 \\
\pm 5.55\end{array}$ \\
\hline $\begin{array}{l}\text { sigmoid colon } \\
\text { SCS }\end{array}$ & $\begin{array}{l}78.23 \\
\pm 15.65\end{array}$ & $\begin{array}{l}59.69 \\
\pm 17.62\end{array}$ & $\begin{array}{l}48.49 \\
\pm 14.13\end{array}$ & $\begin{array}{l}39.94 \\
\pm 14.20\end{array}$ & $\begin{array}{l}27.23 \\
\pm 13.19\end{array}$ & $\begin{array}{l}4.51 \\
\pm 4.72\end{array}$ & $\begin{array}{l}1.94 \\
\pm 2.03\end{array}$ & $\begin{array}{l}38.9 \\
\pm 4.09\end{array}$ \\
\hline $\begin{array}{l}\text { sigmoid colon difference } \\
\text { (BOX- SCS) }\end{array}$ & $\begin{array}{l}11,98 \\
\pm 10,62\end{array}$ & $\begin{array}{l}26.67 \\
\pm 16.74\end{array}$ & $\begin{array}{l}34.12 \\
\pm 15.91\end{array}$ & $\begin{array}{l}38.67 \\
\pm 16.69\end{array}$ & $\begin{array}{l}45.43 \\
\pm 17.32\end{array}$ & $\begin{array}{l}5.03 \\
\pm 4.95\end{array}$ & $\begin{array}{l}2.42 \\
\pm 3.20\end{array}$ & $\begin{array}{l}9.08 \\
\pm 3.44\end{array}$ \\
\hline $\begin{array}{l}\text { bladder } \\
\text { BOX }\end{array}$ & $\begin{array}{l}98.90 \\
\pm 3.11\end{array}$ & $\begin{array}{l}98.52 \\
\pm 4.18\end{array}$ & $\begin{array}{l}98.22 \\
\pm 5.05\end{array}$ & $\begin{array}{l}96.78 \\
\pm 5.59\end{array}$ & $\begin{array}{l}94.92 \\
\pm 6.57\end{array}$ & $\begin{array}{l}82.26 \\
\pm 15.33\end{array}$ & $\begin{array}{l}64.89 \\
\pm 17.23\end{array}$ & $\begin{array}{l}64.47 \\
\pm 4.74\end{array}$ \\
\hline $\begin{array}{l}\text { bladder } \\
\text { SCS }\end{array}$ & $\begin{array}{l}98.18 \\
\pm 4.06\end{array}$ & $\begin{array}{l}94.74 \\
\pm 7.72\end{array}$ & $\begin{array}{l}91.22 \\
\pm 9.48\end{array}$ & $\begin{array}{l}86.36 \\
\pm 11.43\end{array}$ & $\begin{array}{l}80.71 \\
\pm 12.83\end{array}$ & $\begin{array}{l}72.17 \\
\pm 15.03\end{array}$ & $\begin{array}{l}60.94 \\
\pm 17.28\end{array}$ & $\begin{array}{l}61.67 \\
\pm 5.97\end{array}$ \\
\hline $\begin{array}{l}\text { bladder } \\
\text { difference (BOX- SCS) }\end{array}$ & $\begin{array}{l}0.72 \\
\pm 1.18\end{array}$ & $\begin{array}{l}3.79 \\
\pm 6.06\end{array}$ & $\begin{array}{l}7.00 \\
\pm 7.59\end{array}$ & $\begin{array}{l}10.41 \\
\pm 8.93\end{array}$ & $\begin{array}{l}14.21 \\
\pm 10.19\end{array}$ & $\begin{array}{l}10.10 \\
\pm 5.64\end{array}$ & $\begin{array}{l}3.95 \\
\pm 2.77\end{array}$ & $\begin{array}{l}2.8 \\
\pm 1.98\end{array}$ \\
\hline
\end{tabular}

fer to the big fields of gantries $0^{\circ}$ and $180^{\circ}$, whereas, the small fields as well as fields from other directions remain unchanged.

\section{Methods}

\section{The SCS with individual shielding blocks}

The region between iliac nodes, not included in PTV1, can be shielded from $0^{\circ}$ and $180^{\circ}$ with individual shielding blocks. The shielding blocks for both fields are made according to the PTV1. Eight $\mathrm{cm}$ thick shielding blocks are made of Wood alloy. At our department we are using the individual shielding blocks at Elekta Synergy Platform linear accelerator.

\section{The SCS with the off centre fields splitting}

Shielding can be preformed also with multi-leaf collimator (MLC) as presented in the
Figure 1. At our department the majority of the patients with locally advanced and high risk localized prostate tumour are scheduled to be treated at linear accelerator Varian 2100 CD, where individual shielding blocks are not in use, and, therefore, we need to perform SCS with multi-leaf collimator.

In order to shield the part in the middle of the field, the fields have to be split across the region of shielding. The collimator of the field should be turned so the MLC-s can slide perpendicular to the field cut. Pre-optimized conformly shaped $0^{\circ}$ and $180^{\circ}$ fields are duplicated and shaped in the left and the right part. The outer margins of the duplicated fields remain unchanged, but the margins at left - right junctions have to be shaped according to the PTV1 as presented in the Figure 1. After the shaping, the irradiation times of left and right parts are set to the same optimal irradiation time.

In case the fields are split in two parts through the centre (half beam blocks-HBB), 


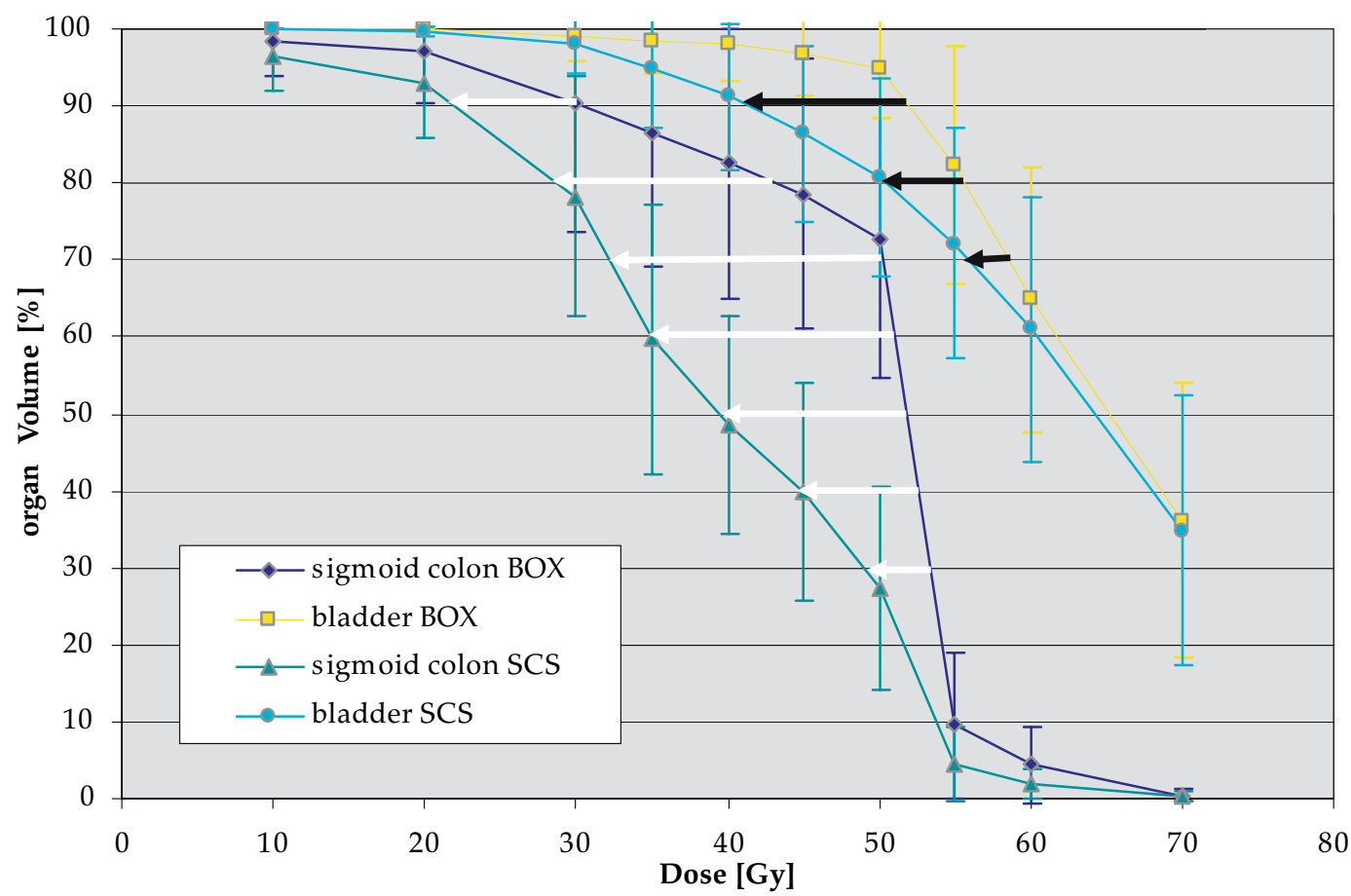

Figure 2. Cumulative dose volume histograms (DVH) for sigmoid colon and bladder for box alone and sigmoid colon shielding (SCS) technique. The dose on sigmoid colon and bladder in high risk prostate cancer irradiation were evaluated with the DVH. The cumulative DVH for box alone and for SCS technique were analyzed in 10 cases. In every single case, both sigmoid colon and bladder were spared with SCS. The figure presents the average DVH for sigmoid colon and bladder for both techniques. The arrows indicate the dose reduction on the parts of organs when applying SCS.

both beams $0^{\circ}$ and $180^{\circ}$ are split in the same central plane. In such case fields from both directions $\left(0^{\circ}\right.$ and $\left.180^{\circ}\right)$ contribute to the potential cold or hot spots at the field junction plane. Therefore, we are proposing the "off centre field splitting". The experience shows that $0.5 \mathrm{~cm}$ off centre splitting can still support the appropriate shielding of the part not included in PTV1. The split for the two fields $\left(0^{\circ}\right.$ and $\left.180^{\circ}\right)$ should be at left and right to the central line, so there is a 1 $\mathrm{cm}$ gap between the 0 and 180 split planes. With this kind of splitting the cold and hot spots are blurred compared to the splitting in the one plane since the contribution to the cold and hot spots at split planes arises from one side only. Therefore, the possible inaccuracy in delivered dose is lowered.

\section{The evaluation of the SCS}

The SCS technique was evaluated in group of 10 consecutive patients that received radical radiotherapy treatment for locally advanced and high risk localized prostate tumour. To plan the treatment, CT images were taken with Philips MX 8000 and used with CMS XIO planning system. In all patients both treatment techniques were planned: box alone and SCS. The techniques were evaluated with dose volume histograms (DVH) comparison. We were checking and comparing the PTV coverage and organs at risk exposure (rectum, anus, sigmoid colon, bladder, penile bulb, acetabulum, small intestine). The detailed analysis was carried out for sigmoid colon and bladder, since in all the cases the dif- 


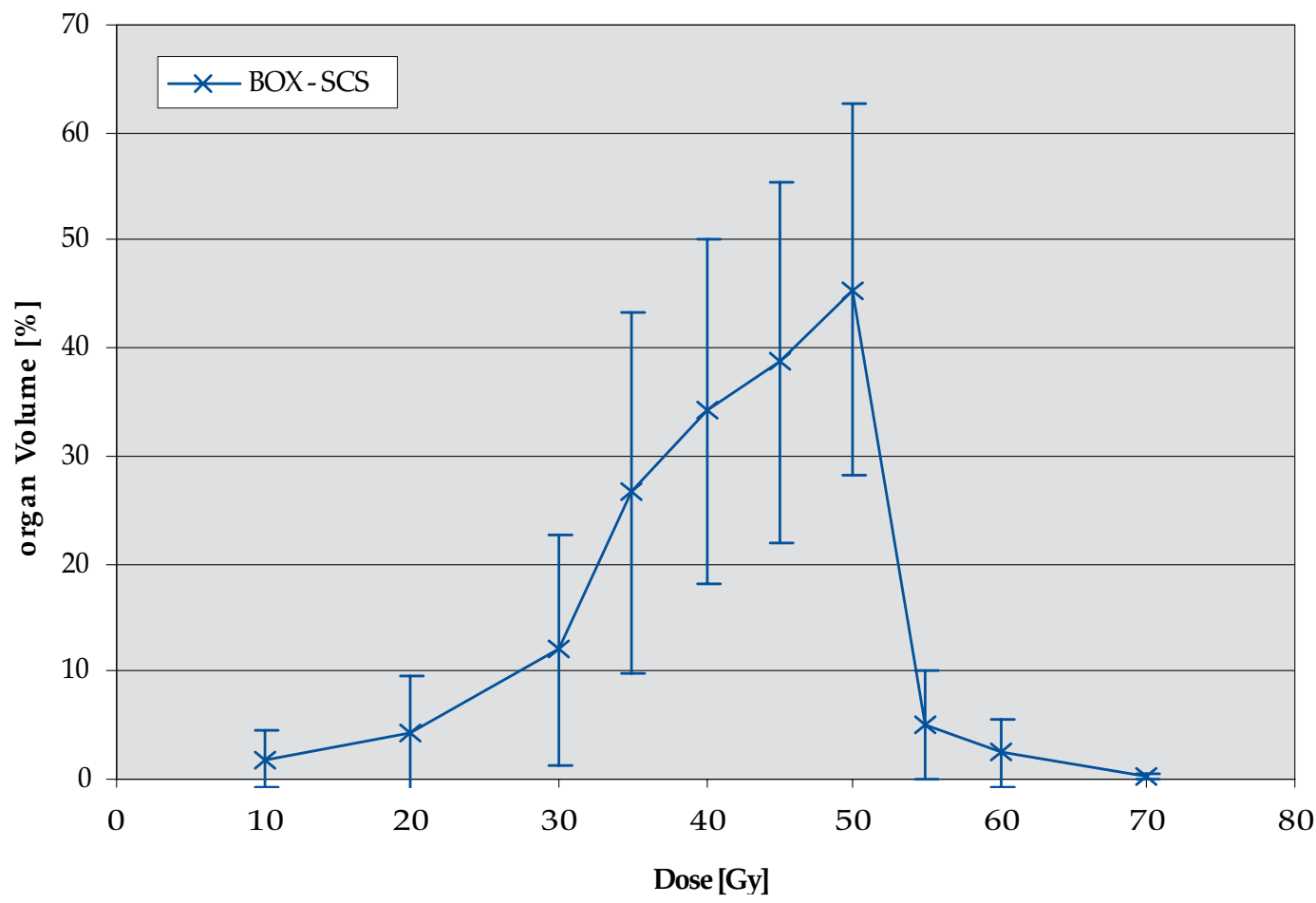

Figure 3. The average difference in cumulative DVH for sigmoid colon between box alone and SCS. Using the SCS technique at WPI of high risk prostate irradiation the inclusion of the sigmoid colon the irradiation region is reduced. Around 30-40\% of the whole sigmoid colon that would be irradiated with 35 to 50 Gy with box technique is spared with sigmoid colon shielding.

ference in DVH was the greatest for the two organs. The differences in V10, V20, V30, V35, V40, V45, V50, V55, V60, V70 and in mean dose for sigmoid colon and for bladder were calculated for every patient for box alone and SCS technique. Since the number of patients is still small only the average difference in DVH parameters and their standard deviation is presented and commented.

\section{Results}

Applying the Sigmoid Colon Shielding (SCS) technique in the WPI the dose to sigmoid colon and bladder is importantly reduced, at the unchanged coverage of PTV.
Since the WPI represents two thirds of the radiotherapy for locally advanced and high risk localized prostate tumour, any changes that are introduced in planning WPI express notably in the cumulative treatment plan. The comparison of the average DVH of box alone and SCS technique is presented in the Figure 2 and Table 1.

The general idea of the average DVH, presented in the Figure 2 and Table 1, holds also for every single case observed. As can be seen from DVH comparison, applying SCS a part of the sigmoid colon and bladder can be spared. The most obvious is saving of the part of the sigmoid colon that would receive $35 \mathrm{~Gy}$ to $50 \mathrm{~Gy}$ with boxalone, whereas there is almost no difference in the irradiation of the parts of sig- 


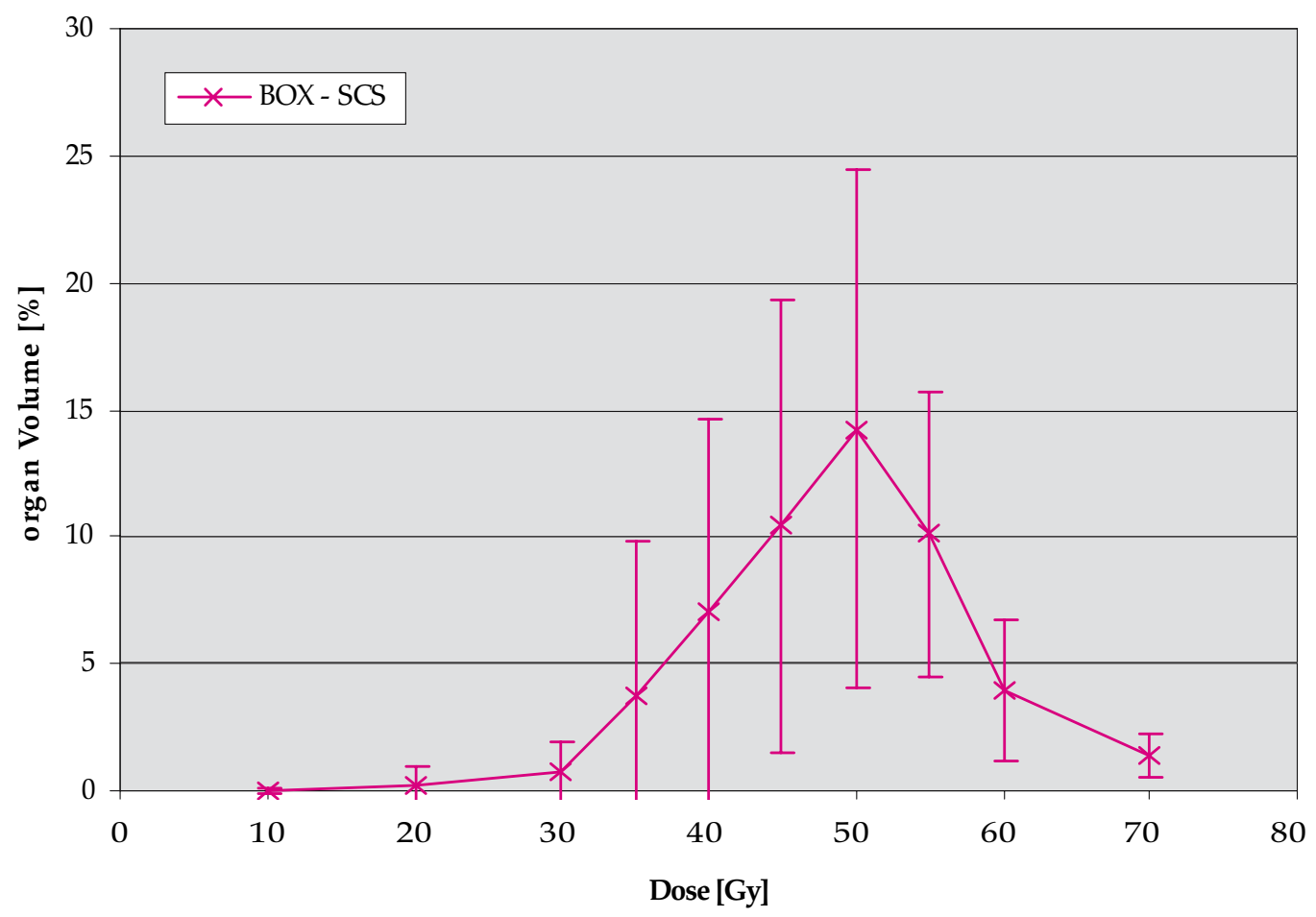

Figure 4. The average difference in cumulative DVH for bladder between box alone and SCS. Around 10\% of the bladder that would be irradiated with 45 to 55 Gy with box alone is spared applying SCS technique. Although, compared to the sigmoid colon) the smaller part of the bladder is spared, the higher doses are lowered.

moid colon with smaller or higher doses. The bladder sparing is not as obviously expressed than the sigmoid colon sparing, but the proportion of the bladder that would receive higher doses is reduced.

Since the parts of the sigmoid colon and bladder that are not shielded are in SCS technique are irradiated the same way as with box alone, the shift of DVH curves holds also the relevant information of the dose reduction on sigmoid colon and bladder due to the shielded region sparing (indicated with arrows in Figure 2). The average dose reduction on the majority (70\%) of the sigmoid colon reduction can be almost 20 Gy (from 50 Gy to almost 30 Gy). Applying SCS $90 \%$ of the bladder can be irradiated with approximately 10 Gy smaller doses (dose is reduced from 55
Gy to 45 Gy), as can be seen following the black arrow.

Figure 3 presents the average difference in DVH of sigmoid colon between box alone and SCS technique. Form 30 to $45 \%$ or in some cases even up to $55 \%$ of the sigmoid colon irradiated at doses from 35 to $50 \mathrm{~Gy}$ is spared applying SCS. The average sparing of the sigmoid colon is in spite of small patient number (10) significant, even 2.5 times as big as the standard deviation.

With SCS technique also bladder is spared. The benefit for the bladder can be seen in Figure 4, that presents the average difference in DVH for bladder between box alone and SCS technique, can be seen that around $10 \%$ of the bladder that receives 45 55 Gy with box alone can be spared with SCS technique. 
It was also observed in DVH that small intestine does a bit better with SCS, but the detailed analysis was not preformed.

\section{Discussion}

Applying the SCS technique instead box alone in WPI the sigmoid colon and bladder can be spared at the unchanged PTV coverage. We assume that with the decreased dose on organs at risk also the toxicity of the irradiation is reduced. ${ }^{7,9} \mathrm{We}$ tend to prepare the report on lowered toxicity due to SCS implementation.

We have in mind that in order to compare dose volume histograms resulting from different applied techniques one has to be extremely careful, since completely different techniques can arise in same DVH. In our cases we are confident to compare DVH, since we keep in mind that most of the technique and therefore the dose delivered to the majority of the irradiated volume remained unchanged, and all the differences in DVH arise from the shielding part. We are presenting the average difference in DVH between the SCS technique and box alone, but also, in every single case, there was a beneficial difference observed in favour of SCS technique. The beneficial changes in DVH were, dependent on the patient anatomy, expressed in the different parts of the DVH. This was leading to the higher standard deviation of the average DVH difference. With higher number of patients, we expect that the difference between techniques would be more accurately expressed and therefore the presented benefit of applying the SCS technique would be greater than presented.
We also tend to improve the bladder sparing. Our current advices to the patients lead us to the bladders of volumes around $100 \mathrm{~cm}^{3}$. We assume that with bladders filled up to $200 \mathrm{~cm}^{3}$ the dose on bladder decrease. ${ }^{8}$ Combined with SCS technique the dose decrease would be even more important. Therefore, we are preparing to change our current advice to patient to result in bladders of volumes around $200 \mathrm{~cm}^{3}$.

\section{Conclusions}

Sparing the region between the iliac nodes is beneficial since the dose delivered to the organs at risk that are partly included in the mentioned area (the sigmoid colon, and bladder) can be importantly reduced. In our analysis we evaluated the reduction of the dose delivered to the sigmoid colon and bladder with the implementation of the SCS technique. Even with the small number of analysed cases, at the unchanged PTV coverage, the dose reduction on OR is evident. Due to the dose reduction on the organs at risk, the reduction of the irradiation toxicity is also expected. Therefore, the toxicity will be monitored and reported later on.

\section{Acknowledgement}

The improvement of the well established and common techniques can only be carried out with coordinate cooperation of all the workers involved in the process. Therefore I would like to thank to all the coworkers and the crew that are working hard in order to improve the treatment quality. 


\section{References}

1. Roach M 3rd, DeSilvio M, Lawton C, Uhl V, Machtay M, Seider MJ, et al. Phase III trial comparing whole-pelvic versus prostate-only radiotherapy and neoadjuvant versus adjuvant combined androgen suppression: Radiation Therapy Oncology Group 9413. J Clin Oncol 2003; 21: 1904-11.

2. Jani A B, Su A, Milano MT. Intensity -modulated versus conventional pelvic radiotherapy for prostate cancer: analysis of acute toxicity. Urology 2006; 67: 147-51.

3. Peeters ST, Hoogeman MS, Heemsbergen WD, Hart AA, Koper PC, Lebesque JV. Rectal bleeding, fecal incontinence, and high stool frequency after conformal radiotherapy for prostate cancer: Normal tissue complication probability modeling. Int J Radiat Oncol Biol Phys 2006; 66: 11-9.

4. Skala M, Rosewall T, Dawson L, Divanbeigi L, Lockwood G, Thomas C, et. al. Patient-assessed late toxicity rates and principal component analysis after image-guided radiation therapy for prostate cancer. Int J Radiat Oncol Biol Phys 2007; 68: 690-8.

5. Wawrovschek F, Vogt $\mathrm{H}$, Wengenmair $\mathrm{H}$, Weckermann D, Hamm M, Keil M, et al. Prostate lymphoscintigraphy and radioguided surgery for sentinel lymph node identification in prostate cancer. Technique and resultes of the first 350 cases. Urol Int 2003; 70: 303-10.

6. De Meerleer GO, Villeirs GM, Vakaet L, Delrue LJ, De Neve WJ. The incidence of inclusion of the sigmoid colon and small bowel in the planning target volume in radiotherapy for prostate cancer. Strahlenther Oncol 2004, 9: 573-81.

7. Fonteyene V, De Neve W, Villeirs G, De Wagter C, De Meerleer G. Late radiotherapy-induced lower intestinal toxicity (RILIT) of intensity modulated radiotherapy for prostate cancer: the need for adapting the toxicity scales and the appearance of the sigmoid colon as co-responsible organ for lower intestinal toxicity. Radiother Oncol 2007; 84: 156-63.

8. Pinkawa M, Fischendick K, Asadpour B, Gagel B, Piroth MD, Eble MJ. Low-grade toxicity after conformal radiation therapy for prostate cancer - impact of bladder volume. Int J Radiat Oncol Biol Phys 2006; 64: 835-41.
9. Cheung MR, Tucker SL, Dong L, de Crevoisier R, Lee AK, Frank $S$, et al. Investigation of bladder dose and volume factors influencing late urinary toxicity after external beam radiotherapy for prostate cancer. Int J Radiat Oncol Biol Phys 2007; 67: 1059-65.

10. Wang-Chesebro A, Xia P, Coleman J, Akazawa C, Roach M 3rd. Intensity-modulated radiotherapy improves lymph node coverage and dose to critical structures compared with three-dimensional conformal radiation therapy in clinically localized prostate cancer. Int J Radiat Oncol Biol Phys 2006; 66: 654-62. 